\title{
Part perception in infancy: Sensitivity to the short-cut rule
}

\author{
Ramesh S. Bhatt, Angela Hayden, Ashley Kangas, \\ Nicole Zieber, AND JANE E. JoSEPH \\ University of Kentucky, Lexington, Kentucky
}

\begin{abstract}
Research indicates that object perception involves the decomposition of images into parts. A critical principle that governs part decomposition by adults is the short-cut rule, which states that, all else being equal, the visual system parses objects using the shortest possible cuts. We examined whether 6.5-month-olds' parsing of images also follows the short-cut rule. Infants in the experimental conditions were habituated to cross shapes and then tested for their preference between segregated patterns produced using long cuts versus short cuts. Infants in the control conditions were directly tested with the segregated patterns. Infants in the experimental conditions exhibited a greater novelty preference for the long-cut over the short-cut patterns than did those in the control conditions, thereby indicating that they are more likely to segregate cross shapes using short cuts rather than long cuts. This sensitivity to the short-cut rule was evident when two alternative parameters, part area and protrusion, were controlled in Experiments 1 and 2, respectively. Thus, a critical principle that governs part segregation in adulthood is operational by 6.5 months of age.
\end{abstract}

A considerable amount of theoretical and empirical effort has been devoted to the study of various aspects of object perception in infancy. For instance, many studies have examined how portions of objects that are segregated by occlusion are combined into holistic objects on the basis of principles such as common movement and feature similarities (e.g., Arterberry, 2001; Bremner, 2007; Johnson, 2009; Johnson \& Aslin, 1996; Johnson \& Slater, 2007; Kavšek, 2004; Kellman \& Spelke, 1983). Other research has examined how infants establish boundaries between objects using such factors as feature differences and edge properties (e.g., Needham, 2001; Needham \& Baillargeon, 1998). There is also a sizeable literature on infants' use of grouping principles to organize local elements into global percepts (for a review, see Quinn, Bhatt, \& Hayden, 2008). On the basis of these and other such efforts, much is known about the processes that underlie the grouping of parts into holistic objects and the global separation of objects. However, little is known about the segregation of objects into parts, which is also an important aspect of object perception. The present experiments address this issue by examining whether 6.5-month-old infants utilize the short-cut rule, a principle that has been shown to affect part segregation in adulthood.

The lack of knowledge about part segregation in infancy is noteworthy because this function plays a highly significant role in object recognition (e.g., Biederman, 1987; Feldman \& Singh, 2005; Hoffman \& Richards, 1984; Marr \& Nishihara, 1978; Palmer, 1977; Singh \& Hoffman, 2001). For instance, Marr and Nishihara suggested that, when exposed to an image of an object, the adult visual system extracts the generalized cone shape parts that comprise the image and then generates a representation of the object based on these parts and the spatial relations among them. Similarly, Biederman's model assumes that the adult visual system decomposes images into volumetric shape parts and uses these parts and their spatial relations to create representations that allow us to recognize and interact with objects. In addition to playing a central role in these influential models, part perception has been tied to a number of critical visual functions, such as figure-ground assignment, shape similarity, visual search, allocation of attention, and memory for shapes (for a review, see Singh \& Hoffman, 2001).

Given the significant role of part processing in human perception, one question that arises is how we compute parts from images. Some researchers have suggested that the visual system utilizes a limited set of computational principles or rules to determine parts (e.g., Hoffman \& Singh, 1997; Siddiqi, Tresness, \& Kimia, 1996; Singh \& Hoffman, 2001; Xu \& Singh, 2002). Thus, according to this view, one goal of research should be to specify and understand the rules that determine the part decomposition processes.

In a series of theoretical and empirical articles, Hoffman, Singh, and their colleagues have developed a set of principles that the adult visual system applies to generate parts from images (e.g., Hoffman \& Richards, 1984; Hoffman \& Singh, 1997; Singh \& Hoffman, 2001). For instance, one principle that is widely accepted is the minima rule, which refers to the role of concavities (local minima of curvature) in part perception. Hoffman and Richards

R.S.Bhatt, rbhatt@email.uky.edu 
noted that there is a concave inflexion where a part intersects the rest of an object. Thus, according to the minima rule, boundaries between parts are signaled by minima of curvature or concavities.

However, Singh, Seyranian, and Hoffman (1999; see also Hoffman \& Singh, 1997; Siddiqi et al., 1996; Singh \& Hoffman, 2001) noted that the minima rule by itself is not sufficient to determine parts. Whereas this rule can be used to identify part boundaries, it does not specify the parts themselves. That is, it does not indicate how concavities might be connected to parse objects. Also, sometimes part boundaries are not at concavities and, conversely, not all concavities signal part boundaries. Thus, other principles besides the minima rule itself are needed to specify how parts are derived from images.

Singh et al. (1999) proposed the "short-cut" rule as a principle that builds upon the minima rule for part segregation. The short-cut rule states that "other things being equal, human vision prefers to use the shortest possible cuts to parse silhouettes" (p. 636). Singh et al. suggested that the short-cut rule is motivated by the geometry of 3-D intersection: When a narrower cylinder completely intersects (i.e., extends through) a wider cylinder, the concave creases that result at the intersections encircle the smaller cylinder and not the larger cylinder (see Figure 1). Thus, following the minima rule, the part cuts should be along the concave creases, which would be the shorter cuts because they encircle the narrower rather than the wider cylinder.

Singh et al. (1999) found that adults' parsing of crossand L-shaped images was in accord with the short-cut rule. De Winter and Wagemans (2006) found that the use of the short-cut rule was evident even when adults parsed more complex stimuli than the ones studied by Singh et al.- namely, the Snodgrass and Vanderwart (1980) line drawings. These findings provide empirical support for the theoretical derivation of the short-cut rule by Singh et al. and indicate that adults use this rule while parsing objects.

Very little is known about part perception in infancy. There is evidence that infants are sensitive to concavities. Arterberry and Yonas $(1988,2000)$ found that infants are sensitive to the presence versus absence of concavities. Bhatt, Hayden, Reed, Bertin, and Joseph (2006) found that, like adults, infants detect concavities among convexities more readily than vice versa. Presumably, infants' sensitivity to concavities reflects concavities' importance as potential part boundaries. There is also some research suggesting that infants are sensitive to some aspects of parts themselves: Hayden and Bhatt (2008) found that infants as young as 5 months of age attend more to the shape of parts than to the shape of cavities. Also, research by Quinn and colleagues suggests that infants parse overlapping outline shapes systematically on the basis of gestalt rules such as good continuation and also on the basis of prior experience with segments of the shapes (Quinn, Brown, \& Streppa, 1997; Quinn \& Schyns, 2003; Quinn, Schyns, $\&$ Goldstone, 2006). Although these studies suggest that at least some aspects of part perception in adulthood are evident in infancy, no study has examined whether infants

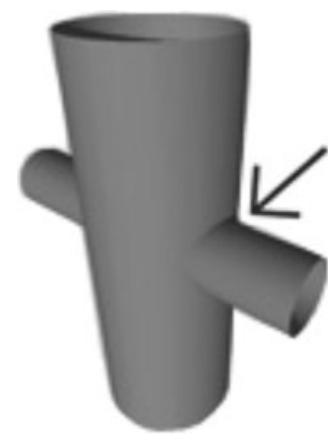

Figure 1. Illustration of the short-cut rule (adapted from Singh $\&$ Hoffman, 2001). As indicated by the arrow in the figure, when a narrower cylinder extends through a wider cylinder, the concave creases that result at the intersections encircle the smaller cylinder and not the larger cylinder. If parts are segregated at concavities in accord with the minima rule, then the part cuts would be around the narrow cylinder (where there is concavity) rather than around the larger cylinder (where concavity is absent). Thus, all else being equal, parts are segregated using the shortest possible cuts, which is the short-cut rule.

are sensitive to the perceptual segregation principles that drive part perception in adulthood.

The goal of the present study was to address this gap in the literature. Specifically, we examined infants' sensitivity to the short-cut rule by testing whether infants find partitions of cross-shaped images made on the basis of the short-cut rule to be more appropriate than alternative segregations. If so, then it would suggest that a critical rule that adults use to segregate objects is already functional early in life.

\section{EXPERIMENT 1}

In this experiment, we examined whether infants segregate shapes with multiple parts in accord with the short-cut rule. Singh et al. (1999) tested adults with images of the sort shown in Figure 2A. Note that the segregation in Figure 2B involves shorter cuts than the segregation in Figure 2C; therefore, the short-cut rule predicts the former to be the more appropriate parsing of the image in Figure 2A. Consistent with this prediction, Singh et al. found that adults segregate in accord with the short-cut rule (i.e., as in Figure 2B rather than as in Figure 2C). In the present experiment, we examined whether 6.5 -month-old infants also find the pattern like the one shown in Figure 2B to be a more appropriate parsing of cross-shaped images than the example shown in Figure 2C. If so, then it would suggest that infants of this age are sensitive to the short-cut rule.

We chose to study 6.5-month-olds in this initial investigation of infants' sensitivity to part decomposition rules because some researchers have suggested that younger infants may not be sensitive to edge-based properties of objects (e.g., Arterberry, 2001; Kellman, 1996; Kellman \& Arterberry, 1998; Kellman \& Shipley, 1991; but see Bhatt et al., 2006; Hayden, Bhatt, \& Quinn, 2008). Specifically, Kellman and colleagues contrasted two processes, a "primitive" edge-insensitive process that is presumed to 


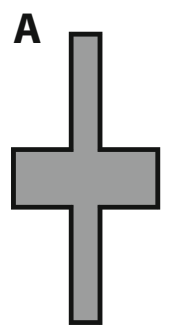

Habituation

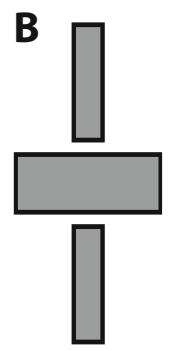

Short-Cut Test

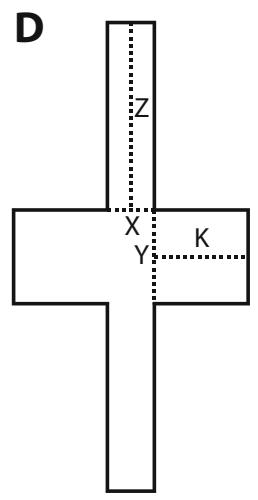

Figure 2. Examples of the stimuli used in Experiment 1. Infants were habituated to stimuli of the sort shown in Figure $2 \mathrm{~A}$ and were tested with stimuli of the sort shown in Figures 2B and 2C. Two such sets of stimuli were used: those in which the short cuts were horizontal whereas the long cuts were vertical (as in Figures 2A-2C) and those in which the short cuts were vertical and the long cuts were horizontal (i.e., $90^{\circ}$-rotated versions of the stimuli in Figures 2A-2C). Figure 2D depicts the parameters used to characterize the stimuli (adapted from Singh, Seyranian, \& Hoffman, 1999): $X$ = length of the short cut; $Y=$ length of the long cut; $Z=$ length (protrusion) of the part associated with the short cut; $K=$ length (protrusion) of the part associated with the long cut. Please see the text for further details.

be operational from birth and that utilizes common motion information, and a maturationally determined "rich" edge-sensitive process that becomes available beginning at around 6-7 months of age and responds to good continuation information. Given that the short-cut rule relies on part-cuts at inflexion points on edges, we thought it would be best to start examination of infants' sensitivity to this rule at an age when infants are sensitive to edge-based cues.

Singh et al. (1999) proposed that cross-shaped images can be characterized using three parameters (see Figure 2D): the relative area of parts resulting from the short versus long cuts ( $\mathrm{XZ}$ vs. YK), the protrusion ratios of parts (i.e., the length of a part divided by the extent of its base; here, $\mathrm{Z} / \mathrm{X}$ vs. K/Y), and the cut lengths (X vs. Y). They systematically varied images on the basis of these parameters and found that adults' parsing was determined mostly by the cut length (i.e., adults chose the shorter cuts to be more appropriate); the effects of the other two parameters were not systematic and were seen only in limited cases.
On the basis of these data, Singh et al. concluded that the short-cut rule best captured adults' part segregation.

Because it was not possible to test infants in the present study on the whole set of stimuli used by Singh et al. (1999), we chose to equate stimuli in terms of the part area and protrusion ratio parameters to examine whether, under those conditions, infants' parsing is sensitive to the short-cut rule. In other words, we tested whether infants' behavior would be consistent with the short-cut rule with stimuli in which the areas and the protrusion ratios of the parts created were the same regardless of whether the cuts were short or long. In Experiment 1, we equated the area of the parts that would result from short versus long cuts.

We employed the commonly used infant-control habituation procedure in this study (e.g., Bhatt, Bertin, Hayden, \& Reed, 2005; Hayden et al., 2008; Horowitz, Paden, Bhana, \& Self, 1972). In this procedure, infants are repeatedly exposed to a pattern until they habituate to it. Then, infants are tested with paired presentations of two stimuli, one of which is either the familiar stimulus or a pattern that is similar to it, whereas the other is a novel pattern. Infants typically exhibit a preference for the novel pattern, or at least a greater preference for the novel pattern in comparison with a control group that is directly tested with the novel and familiar patterns without any habituation. If this occurs, then it indicates that they found the familiar pattern to be more similar to the habituation stimulus than the novel pattern. In the present experiments, infants in the experimental conditions were habituated to cross-shaped patterns and tested with part segregated images that were generated with either short cuts (short-cut patterns) or long cuts (long-cut patterns; see Figure 2). Infants in the control conditions were directly tested with the short-cut and long-cut patterns without any habituation to assess any "spontaneous" preference that infants might have for one of these patterns. If infants' preference for the long-cut over the short-cut patterns is greater in the experimental than in the control condition, then it was inferred that the infants found the short-cut patterns to be the more appropriate segregation of the cross shapes to which they were habituated. Note that the assumption here is that infants' performance during the test is driven by the habituation experience in the experimental condition, whereas it is driven by spontaneous preferences between the test stimuli in the control conditions because infants in the latter conditions were directly tested without any habituation.

\section{Method}

Participants. Twenty-four 6.5-month-olds participated (mean age $=210.25$ days; $S D=8.31$ ). They were predominantly Caucasians from middle-class families. Data from an additional 7 infants were not included in this analysis because of sibling interference $(n=3)$, preferential looking (greater than $90 \%$ looking toward a stimulus; $n=1)$, failure to look on a test trial $(n=1)$, and crying $(n=2)$.

Stimuli. The stimulus patterns used are depicted in Figure 2. Each infant was habituated to a cross pattern in which the areas of the parts that would be derived by long cuts $(\mathrm{Y})$ versus short cuts $(\mathrm{X})$ were equated. In Figure 2A, for example, the area XZ $\left(6.45 \mathrm{~cm}^{2}\right)$ was equal 
to the area YK. Thus, regardless of whether the cross shape is cut along the $\mathrm{X}$ boundaries or the $\mathrm{Y}$ boundaries, the resulting parts are of the same area. The ratio of the short cut $(X=1.27 \mathrm{~cm})$ to the long cut $(\mathrm{Y}=2.54 \mathrm{~cm})$ was .50 . Recall that the protrusion ratio is the length of a part divided by the extent of its base. Here, the protrusion ratio of the thinner parts $(Z / X ; Z=5.08 \mathrm{~cm}, X=1.27 \mathrm{~cm})$ was 4 , whereas that of the thicker parts $(\mathrm{K} / \mathrm{Y} ; \mathrm{K}=2.54 \mathrm{~cm}, \mathrm{Y}=2.54 \mathrm{~cm})$ was 1 . Thus, with the stimuli used in this experiment, the part areas were matched but the protrusion ratios were not. This is because, with cross stimuli of the kind used in this experiment, which were adapted from Singh et al. (1999), it is not possible to match on both parameters while also having a difference between the long- and the short-cut lengths. As described below, the stimuli used in Experiment 2 were matched in terms of protrusion but not in terms of part areas. Overall, the habituation pattern in Experiment 1 subtended $13.37^{\circ} \times 7.00^{\circ}$.

The test stimuli were generated by making two cuts and displacing the resulting parts by $0.63 \mathrm{~cm}$. The cuts were the short ones (X) for the short-cut patterns and the long ones (Y) for the long-cut patterns. Overall, the short-cut pattern subtended $13.37^{\circ} \times 8.61^{\circ}$, whereas the long-cut pattern subtended $14.97^{\circ} \times 7.00^{\circ}$.

In the present experiment, two different versions of cross and test stimuli were used, so that the short cuts and long cuts were equally often vertical and horizontal (i.e., these images were $90^{\circ}$ rotated versions of each other). Also, as in Singh et al. (1999), the stimuli contained dark outlines and gray interiors in order to prevent illusory contours that could result if the stimuli were uniformly gray or black (i.e., the perception that there are two rectangular objects, one occluding the other; see Figure 23 in Singh et al., 1999).

Apparatus. The experiment took place in a darkened chamber in which infants sat about $45 \mathrm{~cm}$ in front of a $45-\mathrm{cm}$ computer monitor. A video camera (mounted on top of the monitor) and a DVD recorder (located outside of the test chamber) were used to record infants' looks.

Procedure. An infant-control habituation procedure was used in this experiment (Horowitz et al., 1972). In the experimental condition $(n=12)$, each habituation and test trial began with the presentation of an attention-getter (a rapidly pulsating green/pink pattern) in the middle of the screen. Once the infant looked at this pattern, the experimenter pressed a key to start the trial. During habituation trials, two identical copies of a cross pattern were presented, one on the left side and the other on the right side of the monitor. The stimuli remained until the infant looked away for $2 \mathrm{sec}$ or until $60 \mathrm{sec}$ had passed. Such trials continued until the infant's mean look duration during three consecutive trials was less than $50 \%$ of the mean look duration during the first three trials. Once the infant was habituated, two 10 -sec test trials followed immediately. During these trials, the short-cut pattern was presented on one side of the monitor and the long-cut pattern was presented on the other side. The left/right location of each pattern on the first trial was counterbalanced across infants. Also, to avoid the influence of any side bias, the left/right location of the patterns was switched from the first to the second trial. Moreover, as described above, half of the infants were habituated and tested with images in which the short cuts were vertical, and the other half with images in which the short cuts were horizontal.

A separate group of infants (control condition; $n=12$ ) did not go through the habituation process but were directly tested on the test stimuli to document the spontaneous preference, if any, for one test stimulus over the other. This group allowed us to contrast the spontaneous preference between the long-cut versus short-cut patterns against the preference exhibited after habituation to the cross shape in the experimental condition. If infants segregate the cross shape that they are exposed to during habituation in accord with the shortcut rule, then they should show a greater preference for the novel long-cut pattern after habituation than they would have as the result of any spontaneous preference they might have for this pattern. In other words, if infants are sensitive to the short-cut rule, then their preference for the long-cut pattern over the short-cut pattern should be greater in the experimental than in the control condition.

Infants' look durations were coded offline by an observer, who was unaware of the habituation pattern, the locations of the long-cut and short-cut test patterns, and the test condition. Only the infant was visible to the coder. The speed of the video display was reduced to $25 \%$ of normal speed for coding. The performance of 12 randomly chosen participants was coded by another naive experimenter. The Pearson correlation between the two observers' scores was .99.

\section{Results and Discussion}

The results are displayed in Table 1 and Figure 3. As was required by the procedure, infants in the experimental condition exhibited a significant decline in look durations from the first three to the last three trials during habituation $[t(11)=7.55, p<.001]$.

Infants' performance during the test was assessed using a preference score for the long-cut pattern. This measure is analogous to the novelty preference score that is usu-

Table 1

Mean (and Standard Error) of Fixation Duration During Habituation and Test Trials and Percent Preference for Long-Cut Patterns Exhibited During Test Trials in Experiments 1 and 2

\begin{tabular}{|c|c|c|c|c|c|c|c|c|c|c|c|}
\hline \multirow[b]{4}{*}{$N$} & \multirow{2}{*}{\multicolumn{4}{|c|}{ Habituation }} & \multicolumn{7}{|c|}{ Test } \\
\hline & & & & & \multirow{2}{*}{\multicolumn{2}{|c|}{$\begin{array}{c}\text { Duration } \\
\text { of Looks to } \\
\text { Long-Cut } \\
\text { Pattern (sec) }\end{array}$}} & \multirow{2}{*}{\multicolumn{2}{|c|}{$\begin{array}{c}\text { Duration } \\
\text { of Looks to } \\
\text { Short-Cut } \\
\text { Pattern (sec) }\end{array}$}} & \multirow{2}{*}{\multicolumn{3}{|c|}{$\begin{array}{l}\text { Preference } \\
\text { for Long-Cut } \\
\text { Pattern }(\%)\end{array}$}} \\
\hline & \multicolumn{2}{|c|}{$\begin{array}{l}\text { First Three } \\
\text { Habituation } \\
\text { Trials (sec) }\end{array}$} & \multicolumn{2}{|c|}{$\begin{array}{c}\text { Last Three } \\
\text { Habituation } \\
\text { Trials (sec) } \\
\end{array}$} & & & & & & & \\
\hline & $M$ & $S E$ & $M$ & $S E$ & $M$ & $S E$ & $M$ & $S E$ & $M$ & $S E$ & $t$ \\
\hline \multicolumn{12}{|c|}{ Experiment 1} \\
\hline \multicolumn{12}{|c|}{ Experimental Group (Habituation) } \\
\hline 12 & 25.36 & 2.62 & 9.23 & 1.79 & 9.20 & 0.79 & 4.76 & 0.60 & 65.55 & 3.89 & $3.99^{*}$ \\
\hline \multicolumn{12}{|c|}{ Control Group (Spontaneous Preference) } \\
\hline 12 & $\mathrm{n} / \mathrm{a}$ & & $\mathrm{n} / \mathrm{a}$ & & 8.38 & 0.39 & 7.23 & 0.43 & 53.82 & 2.26 & 1.69 \\
\hline \multicolumn{12}{|c|}{ Experiment 2} \\
\hline \multicolumn{12}{|c|}{ Experimental Group (Habituation) } \\
\hline 12 & 20.00 & 3.06 & 8.36 & 1.33 & 7.20 & 0.69 & 7.32 & 0.61 & 49.41 & 3.53 & -0.17 \\
\hline \multicolumn{12}{|c|}{ Control Group (Spontaneous Preference) } \\
\hline 12 & $\mathrm{n} / \mathrm{a}$ & & $\mathrm{n} / \mathrm{a}$ & & 6.56 & 0.47 & 9.78 & 0.56 & 40.29 & 2.99 & $-3.25^{*}$ \\
\hline
\end{tabular}

${ }^{*} p<.05$; significantly different from the chance level of $50 \%$. 


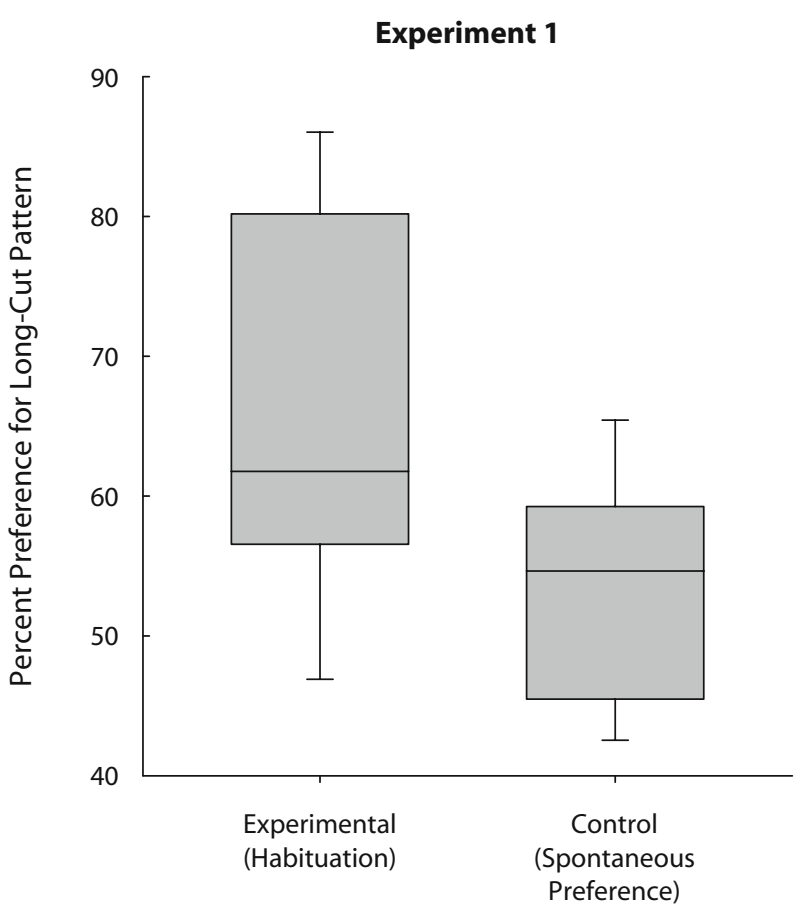

Group

Figure 3. Boxplots of infants' percent preference for the longcut patterns in Experiment 1. The lines inside the boxes indicate the medians, the boxes' boundaries indicate the 25 th and 75 th percentiles, and the whiskers indicate the 10th and 90th percentiles.

ally employed in studies that use the habituation procedure followed by a paired-comparison novelty preference test (e.g., Bhatt et al., 2005; Bhatt et al., 2006; Hayden et al., 2008; Quinn et al., 1997; Quinn et al., 2006). This score was derived by dividing the duration of looking toward the long-cut patterns during the two test trials by the total duration of looking toward both the short-cut and long-cut patterns, and multiplying this ratio by 100 in order to obtain a percent preference score.

Infants' performance indicated sensitivity to the shortcut rule. Infants in the experimental condition displayed a greater mean long-cut preference score than did infants in the spontaneous preference control condition $[t(22)=$ 2.60, $p<.02$ ] (see Table 1 and Figure 3). The difference between the two groups was confirmed by a nonparametric test (Mann-Whitney $U=33, Z=2.25, p<.05$ ).

Moreover, infants' preference score in the experimental condition (Table 1) was significantly greater than the chance level of $50 \%[t(11)=3.99, p<.01]$, which indicates that they found the long-cut pattern (Figure $2 \mathrm{C}$ ) to be more deviant from the habituation pattern (Figure 2A) than the short-cut pattern (Figure 2B). In contrast, infants' score in the control condition was not significantly different from $50 \%[t(11)=1.69, p>.10]$, signifying that infants did not have a spontaneous preference for either pattern. Results of binomial tests supported these findings: 11 out of 12 infants in the experimental group exhibited scores that were greater than $50 \%(p<.01)$, whereas only 8 out of 12 did so in the control group $(p>.38)$.
Thus, infants' performance indicated that they found the short-cut pattern to be the more appropriate segregation of the cross patterns to which they were exposed during habituation. These results suggest that infants' part processing is consistent with the short-cut rule.

Recall that the stimuli used in this experiment were such that the parts produced by the short cuts also protruded more (i.e., had greater protrusion ratios). Some researchers have suggested that protrusion may also affect part segregation: All else being equal, a part is more salient if its protrusion ratio is larger (e.g., Hoffman \& Singh, 1997; Singh et al., 1999). Given this potential contribution of protrusion, it is not possible to unequivocally conclude that infants' performance in Experiment 1 was due to the cut length. Thus, it is important to examine whether infants' performance is in accord with the short-cut rule even under conditions in which part protrusion cannot account for performance. This was the goal of Experiment 2.

\section{EXPERIMENT 2}

In this experiment, we examined whether infants are sensitive to the short-cut rule with images in which the protrusion ratio was equated in the short-cut and long-cut patterns. This allowed us to test whether infants' performance is in accord with the short-cut rule even when protrusion is not a contributing factor. The images we used were similar to those used in Experiment 1, except that the parts associated with the short cut were shortened so that their protrusion ratio was the same as that of the parts associated with the long cuts (see Figure 4).

\section{Method}

Participants. Twenty-four 6.5-month-olds (mean age = 200.88 days; $S D=6.14$ ) participated in this study. Data from an additional 3 infants were excluded due to side bias (greater than $90 \%$ looking toward one side).

Stimuli. The stimuli used in this experiment are shown in Figure 4 . The habituation pattern subtended $9.87^{\circ} \times 7.95^{\circ}$, whereas the short-cut and long-cut test patterns subtended $9.87^{\circ} \times 9.56^{\circ}$ and $11.47^{\circ} \times 7.95^{\circ}$, respectively. They were generated from the ones used in Experiment 1 (Figure 2) by shortening the length of the parts associated with the short cuts (X; see Figure 4D) so that their protrusion ratio of $1(\mathrm{Z} / \mathrm{X} ; \mathrm{Z}=\mathrm{X}=1.27 \mathrm{~cm})$ was the same as the protrusion ratio of the parts associated with long cuts $(\mathrm{K} / \mathrm{Y} ; \mathrm{K}=\mathrm{Y}=$ $2.54 \mathrm{~cm}$ ). Another change from the stimuli used in Experiment 1 was that the dark outlines of the images that surrounded the gray interior were made thinner, because we were concerned that, due to the reduction in protrusion, the smaller parts might not be salient enough for the infants. In all other respects, the stimuli were the same as in Experiment 1.

Note that matching in terms of protrusion led to differences in the areas of the parts generated by short versus long cuts (see Figure 4). Whereas the average area of the resulting parts was the same in both cases (because the number of parts and the overall total area are the same in short-cut and long-cut patterns; see Figure 4), the largest and the smallest parts were in the short-cut pattern. Hoffman and Singh (1997) suggested that part area plays a role in segregation: Their "area" rule states that, all else being equal, a part is more salient the larger it is. However, it is not clear whether the area rule makes a clear prediction about the nature of part segregation in the present experiment. This is because although the largest part was in the short-cut pattern, each of the three part segments in the long-cut pattern is larger than the smaller parts in the short-cut patterns (see 


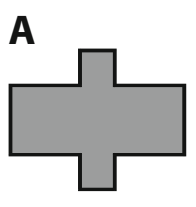

Habituation
B

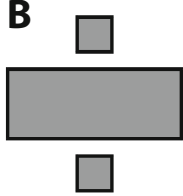

Short-Cut

Test

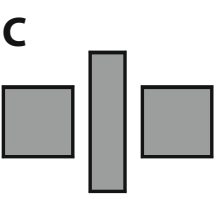

Long-Cut

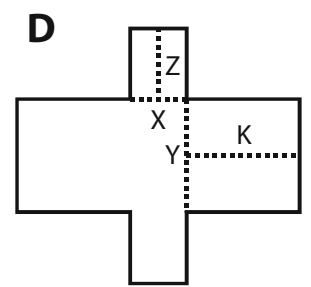

\begin{abstract}
Figure 4. Examples of the stimuli used in Experiment 2. Infants were habituated to stimuli of the sort shown in Figure $4 \mathrm{~A}$ and were tested with stimuli of the sort shown in Figures $4 \mathrm{~B}$ and $4 \mathrm{C}$. Two such sets of stimuli were used: those in which the short cuts were horizontal whereas the long cuts were vertical (as in Figures 4A-4C) and those in which the short cuts were vertical and the long cuts were horizontal (i.e., $\mathbf{9 0}^{\circ}$-rotated versions of the stimuli in Figures $4 \mathrm{~A}-4 \mathrm{C}$ ). The protrusion ratios $(\mathrm{Z} / \mathrm{X}$; see Figure $4 \mathrm{D})$ of the parts associated with the short cuts were matched to the ratios $(K / Y)$ of the parts associated with the long cuts.
\end{abstract}

Figure 4). Thus, the area rule does not make a clear prediction in the present situation. At the same time, the "protrusion" rule also does not make a prediction here because the protrusion ratios of the parts were equated. In contrast, the short-cut rule clearly predicts that the appropriate cuts will be the shorter cuts.

Apparatus and Procedure. The apparatus and procedure were the same as those used in Experiment 1. As in Experiment 1, infants in the experimental condition $(n=12)$ were habituated to cross shapes of the sort shown in Figure 4A and tested with short-cut (Figure 4B) and long-cut (Figure 4C) test patterns. Infants in the control condition $(n=12)$ were directly tested without any habituation to document the degree of spontaneous preference that infants might have for one of the test patterns. Once again, as in Experiment 1, if infants exhibited a greater preference for the long-cut pattern in the experimental than in the control condition, then it would indicate that infants found the short-cut pattern to be the more appropriate segregation of the cross shape.

\section{Results and Discussion}

Infants in the experimental condition exhibited a significant decline in looking from the first three to the last three habituation trials, as required by the procedure (see Table 1) $[t(11)=6.48, p<.001]$.

As in Experiment 1, infants in the experimental condition exhibited a greater mean long-cut preference score during the test than did infants in the spontaneous preference control condition $[t(22)=1.97, p<.03]$ (see Table 1 and Figure 5). The difference between the two groups was confirmed by a nonparametric test (Mann-Whitney $U=$ $38, Z=1.97, p<.05$ ). Unlike in Experiment 1, infants in the control group exhibited a significant preference for the short-cut pattern (as evidenced by a less than $50 \%$ preference for the long-cut pattern) $[t(11)=-3.25, p<.01]$ (Table 1). Thus, infants had a spontaneous preference for the short-cut over the long-cut test pattern. Habituation to the cross pattern allowed infants to overcome this preference, as indicated by the fact that infants in the experimental condition did not exhibit a preference during the test between the long-cut versus short-cut patterns $[t(11)=$ $-0.17, p>.8]$ (and the finding discussed above that infants' score was significantly greater in the experimental than in the control condition; see Table 1 and Figure 5). Results of binomial tests supported these findings: 5 out of 12 infants in the experimental group exhibited scores that were less than 50\% ( $p>.77$; scores less than $50 \%$ indicate preference for the short-cut patterns), whereas 11 out of 12 did so in the control group $(p<.01)$. Thus, the contrast between the appropriate segregation of the cross images afforded by the short-cut pattern versus the unnatural segregation afforded by the long-cut patterns overcame the a priori preference for the short-cut pattern.

In total, the results indicate that infants in this experiment exhibited a greater preference for the long-cut patterns than for the short-cut patterns after habituation to the cross shapes than during a spontaneous preference test (Table 1; Figure 5). Thus, infants' performance was consistent with the short-cut rule even when the protrusion ratios of the short-cut parts matched those of the long-cut parts. This conclusion has to be tempered by the fact that, given the lack of a preference between the short-cut and long-cut patterns during the test in the experimental condition, the results only indirectly indicate that the cross shape would be segregated in accord with the short-cut rule. Despite their indirect nature, however, the results are consistent with those of Experiment 1 in indicating infants' sensitivity to the short-cut rule. 


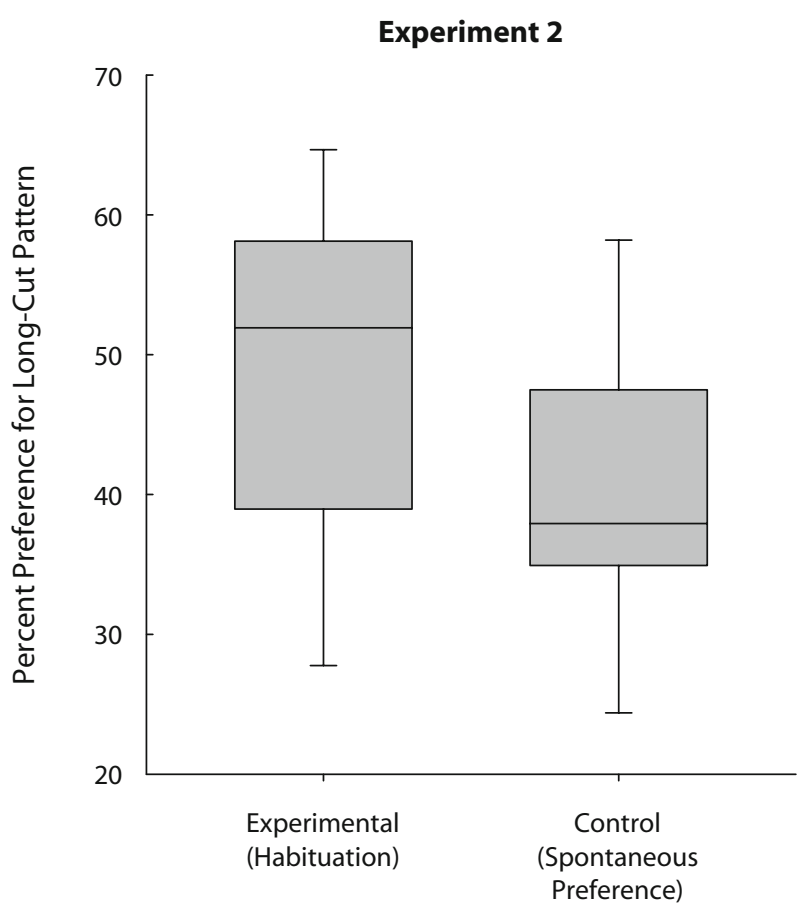

Group

Figure 5. Boxplots of infants' percent preference for the longcut patterns in Experiment 2. The lines inside the boxes indicate the medians, the boxes' boundaries indicate the 25 th and 75 th percentiles, and the whiskers indicate the 10th and 90th percentiles.

\section{GENERAL DISCUSSION}

The present research found that infants as young as 6.5 months of age are sensitive to the short-cut rule of part segregation. Specifically, this research indicated that, like adults in Singh et al. (1999), infants found shorter as opposed to longer cuts to be more appropriate segregations of cross-shaped images. Given the critical nature of part processing to object recognition (e.g., Biederman, 1987; Feldman \& Singh, 2005; Hoffman \& Richards, 1984; Marr \& Nishihara, 1978; Palmer, 1977; Singh \& Hoffman, 2001), this finding suggests that a fundamental principle that governs object recognition in adulthood is available quite early in life.

The present research was based on the theoretical and empirical work of Hoffman, Singh, and their colleagues who proposed the short-cut rule. However, the tendency to find shorter cuts to be more appropriate than longer cuts is also predicted by other models, such as that of Siddiqi et al. (1996), whose proposal that "necks" are a determinant of part-cuts is related to the short-cut rule. According to Siddiqi et al., "a neck is a part-line which is also a local minimum of a diameter of a maximal inscribed circle" (p. 405). The minimum circle diameter is analogous to the shortest cut length. Indeed, in many instances, the Siddiqi et al. notion of "necks" and the Singh et al. (1999) notion of "short-cuts" coincide, and both predict that, all else being equal, shorter cuts would be more likely than longer cuts (De Winter \& Wagemans, 2006). Thus, the short-cut rule as a determinant of part segregation is not necessarily tied to a single theory. Along with this wide theoretical justification, there is also empirical validation of the short-cut rule: In addition to the present research with infants, Singh et al. and De Winter and Wagemans have validated the short-cut rule with adults.

One issue to consider is that the present study may not have documented infants' most typical segregation of the cross shape. Although the data revealed that the infants found the long-cut patterns to be more novel than the short-cut patterns after familiarization to the cross shapes, it is possible that neither test pattern reflects the typical kind of segregation that infants would engage in when confronted with cross shapes. By limiting the choice between two kinds of cuts, we may have missed the possibility that infants might segregate the cross shapes in other ways. We concede that this is a possibility. However, in the absence of an alternative hypothesis about how cross patterns would be segregated by infants, we chose to test predictions of the short-cut rule and the results were consistent with this rule. Also, even if the short-cut rule is not the most salient principle of segregation for infants, and infants typically segregate cross shapes in other ways not reflected in the short-cut stimuli, the present results suggest sensitivity to the short-cut rule. This is because infants' performance followed theoretical predictions based on the short-cut rule and were consistent with adults' use of the short-cut rule.

Another issue concerns the control conditions in which we ascertained a priori preference by testing infants without any familiarization. This is a standard procedure that has been used in a number of studies (e.g., Behl-Chadha, 1996; Bhatt et al., 2005; Bornstein \& Arterberry, 2003; Kovack-Lesh \& Oakes, 2007; Quinn, 1994; Quinn et al., 1997; Quinn, Burke, \& Rush, 1993; Younger \& Fearing, 1999). It could be argued, however, that the fact that infants in the control conditions were tested without any familiarization at all whereas those in the experimental conditions were familiarized may have led to some kind of spurious performance differences. In other words, the act of familiarization itself may have led to changes in preference independent of the object to which infants were familiarized. This could have led to a difference between preferences in the experimental versus control conditions that erroneously appeared to be consistent with the shortcut rule.

Whereas we concede this to be a possibility, we believe that systematic differences that we obtained are unlikely to be due to chance for the following reasons: (1) The patterns of cuts we chose were not arbitrary. The short-cut test stimuli reflect the pattern of cuts predicted by theories that address part segregation in adulthood (e.g., Siddiqi et al., 1996; Singh \& Hoffman, 2001; Singh et al., 1999). (2) Empirical research confirmed theoretical predictions by indicating that adults segregate cross patterns as depicted in the short-cut test stimuli; this makes it likely that infants also engage in a similar kind of segregation (Singh et al., 1999). And (3) in two different experiments, with four different sets of stimuli (vertically and horizontally oriented stimuli in each experiment), infants' preference 
for the long-cut over the short-cut test patterns was significantly greater in the experimental than in the control conditions. Although this could in principle happen just due to the presence versus absence of familiarization in the experimental versus the control conditions rather than to specific exposure to the cross patterns in the former condition, it is more likely that these preferences were based on infants' sensitivity to the short-cut rule.

It should also be noted that, like all such principles in the areas of part segregation and gestalt grouping, the short-cut rule is governed by the "all else being equal" assumption. Thus, the operation of the short-cut rule is a function of other factors that affect part segregation. For instance, each of the two variables that were controlled in the present study by equation (area size in Experiment 1 and protrusion ratio in Experiment 2) may independently govern part segregation in adults (Hoffman \& Singh, 1997; Singh \& Hoffman, 2001; Singh et al., 1999). Also, there are many conditions in which the short-cut rule is overridden. For instance, good continuation can sometimes override the short-cut rule and make a longer cut appear more appropriate than a shorter cut (see Figure 29 in Singh and Hoffman [2001]). Thus, one task of future research is to understand how the various principles that govern part segregation interact with each other.

Future research should also analyze the mechanisms that underlie the development of part segregation before the age tested in the present study. Whereas the present study indicates that at least some basic aspects of part segregation may be available as early as 6.5 months of age, it does not address the prior developmental origins of part segregation. As noted earlier, developmental changes between birth and 6.5 months of age in grouping and other aspects of part aggregation have been documented (e.g., Quinn \& Bhatt, 2005, 2009; Quinn, Bhatt, Brush, Grimes, \& Sharpnack, 2002; Quinn et al., 2008). For instance, grouping on the basis of luminance similarity appears to be available at birth (Farroni, Valenza, Simion, \& Umiltà, 2000), whereas grouping on the basis of shape similarity is not readily available even at 3 to 4 months of age but is available at 6 to 7 months of age (Quinn et al., 2002). Moreover, research suggests that this change may be driven by experience (Quinn \& Bhatt, 2005, 2009). Thus, sensitivity to the short-cut rule may be available at birth (like luminance-based grouping) or it may be something that develops with age and with experience (like shape-based grouping). Future studies will need to examine this issue.

In summary, whereas much is known about grouping processes that lead to holistic object perception, not much is known about the reverse process of part segregation. This is surprising, given that both segregation and grouping play important roles in the object recognition process. Specifically, major theories of object recognition assume that an initial stage of object recognition involves the segregation of images into parts (e.g., Biederman, 1987; Marr \& Nishihara, 1978). Others have suggested that a number of perceptual and cognitive phenomena such as figureground assignment, shape similarity, visual search, allocation of attention, and memory for shapes are due to part segregation and representation processes (for a review, see Singh \& Hoffman, 2001). The present research begins to address this gap in the developmental literature by revealing that the short-cut rule, which is a critical principle that governs part segregation in adulthood, is operational by 6.5 months of age. This finding suggests that at least some basic aspects of the part segregation component of the adult object recognition system are functional quite early in life.

\section{AUTHOR NOTE}

This research was supported by grants from the National Institute of Child Health and Human Development (HD042451, HD052724). We thank the infants and the parents who participated in this study. Address correspondence to R. S. Bhatt, Psychology Department, University of Kentucky, Lexington, KY 40506-0044 (e-mail: rbhatt@email.uky.edu).

\section{REFERENCES}

Arterberry, M. E. (2001). Perceptual unit formation in infancy. In T. F. Shipley \& P. J. Kellman (Eds.), Advances in psychology: Vol. 130. From fragments to objects: Segmentation and grouping in vision (pp. 37-69). New York: Elsevier.

Arterberry, M. E., \& Yonas, A. (1988). Infants' sensitivity to kinetic information for three-dimensional object shape. Perception \& Psychophysics, 44, 1-6.

Arterberry, M. E., \& Yonas, A. (2000). Perception of three-dimensional shape specified by optic flow by 8 -week-old infants. Perception \& Psychophysics, 62, 550-556.

Behl-ChadHa, G. (1996). Basic-level and superordinate-like categorical representations in early infancy. Cognition, 60, 105-141.

Bhatt, R. S., Bertin, E., Hayden, A., \& Reed, A. (2005). Face processing in infancy: Developmental changes in the use of different kinds of relational information. Child Development, 76, 169-181.

Bhatt, R. S., Hayden, A., Reed, A., Bertin, E., \& JosePh, J. (2006) Infants' perception of information along object boundaries: Concavities versus convexities. Journal of Experimental Child Psychology, 94, 91-113.

BIEDERMAN, I. (1987). Recognition-by-components: A theory of human image understanding. Psychological Review, 94, 115-147.

Bornstein, M. H., \& Arterberry, M. E. (2003). Recognition, discrimination and categorization of smiling by 5 -month-old infants. Developmental Science, 6, 585-599.

Bremner, J. G. (2007). Perception and knowledge of the world. In A. Slater \& M. Lewis (Eds.), Introduction to infant development (2nd ed., pp. 137-152). New York: Oxford University Press.

De Winter, J., \& Wagemans, J. (2006). Segmentation of object outlines into parts: A large-scale integrative study. Cognition, 99, 275-325.

Farroni, T., Valenza, E., Simion, F., \& Umiltà, C. (2000). Configural processing at birth: Evidence for perceptual organisation. Perception, 29, 355-372.

Feldman, J., \& Singh, M. (2005). Information along contours and object boundaries. Psychological Review, 112, 243-252.

Hayden, A., \& Bhatt, R. S. (2008, March). Parts, cavities and the development of object representation. Poster presented at the International Conference on Infant Studies, Vancouver, Canada.

Hayden, A., Bhatt, R. S., \& QuinN, P. C. (2008). Perceptual organization based on illusory regions in infancy. Psychonomic Bulletin \& Review, 15, 443-447.

Hoffman, D. D., \& Richards, W. A. (1984). Parts of recognition. Cognition, 18, 65-96.

Hoffman, D. D., \& Singh, M. (1997). Salience of visual parts. Cognition, 63, 29-78.

Horowitz, F. D., Paden, L., Bhana, K., \& Self, P. (1972). An infantcontrol procedure for studying infant visual fixations. Developmental Psychology, 7, 90.

Johnson, S. P. (2009). Developmental origins of object perception. In A. Woodward \& A. Needham (Eds.), Learning and the infant mind (pp. 47-65). New York: Oxford University Press.

Johnson, S. P., \& Aslin, R. N. (1996). Perception of object unity in 
young infants: The roles of motion, depth, and orientation. Cognitive Development, 11, 161-180.

Johnson, S. P., \& SLATER, A. (2007). The development of intelligence in infancy. In A. Slater \& M. Lewis (Eds.), Introduction to infant development (2nd ed., pp. 103-118). New York: Oxford University Press.

KAVŠEK, M. (2004). Infant perception of object unity in static displays. International Journal of Behavioral Development, 28, 538-545.

Kellman, P. J. (1996). The origins of object perception. In R. Gelman \& T. K.-F. Au (Eds.), Perceptual and cognitive development: Handbook of perception and cognition (2nd ed., pp. 3-48). San Diego: Academic Press.

Kellman, P. J., \& Arterberry, M. E. (1998). The cradle of knowledge: Development of perception in infancy. Cambridge, MA: MIT Press.

Kellman, P. J., \& ShiPley, T. F. (1991). A theory of visual interpolation in object perception. Cognitive Psychology, 23, 141-221.

Kellman, P. J., \& Spelke, E. S. (1983). Perception of partly occluded objects in infancy. Cognitive Psychology, 15, 483-524.

Kovack-Lesh, K. A., \& OAKes, L. M. (2007). Hold your horses: How exposure to different items influences infant categorization. Journal of Experimental Child Psychology, 98, 69-93.

MARR, D., \& NishiHARA, H. K. (1978). Representation and recognition of the spatial organization of three-dimensional shapes. Proceedings of the Royal Society B, 200, 269-294.

Needham, A. (2001). Object recognition and object segregation in 4.5-month-old infants. Journal of Experimental Child Psychology, 78, 3-22.

NeedHam, A., \& Baillargeon, R. (1998). Effects of prior experience on 4.5-month-old infants' object segregation. Infant Behavior \& Development, 21, 1-24.

PALMER, S. E. (1977). Hierarchical structure in perceptual representation. Cognitive Psychology, 9, 441-474.

QuinN, P. C. (1994). The categorization of above and below spatial relations by young infants. Child Development, 65, 58-69.

QuinN, P. C., \& BHATT, R. S. (2005). Learning perceptual organization in infancy. Psychological Science, 16, 511-515.

QuinN, P. C., \& BHATT, R. S. (2009). Transfer and scaffolding of perceptual grouping occurs across organizing principles in 3- to 7-month-old infants. Psychological Science, 20, 933-938.

Quinn, P. C., Bhatt, R. S., Brush, D., Grimes, A., \& Sharpnack, H. (2002). Development of form similarity as a Gestalt grouping principle in infancy. Psychological Science, 13, 320-328.
Quinn, P. C., Bhatt, R. S., \& Hayden, A. (2008). What goes with what? Development of perceptual grouping in infancy. In B. H. Ross (Ed.), The psychology of learning and motivation: Advances in research and theory (Vol. 49, pp. 105-146). San Diego: Elsevier.

Quinn, P. C., Brown, C. R., \& Streppa, M. L. (1997). Perceptual organization of complex visual configurations by young infants. Infant Behavior \& Development, 20, 35-46.

QuinN, P. C., Burke, S., \& Rush, A. (1993). Part-whole perception in early infancy: Evidence for perceptual grouping produced by lightness similarity. Infant Behavior \& Development, 16, 19-42.

QuinN, P. C., \& SchYNs, P. G. (2003). What goes up may come down: Perceptual process and knowledge access in the organization of complex visual patterns by young infants. Cognitive Science, 27, 923-935.

QuinN, P. C., Schyns, P. G., \& Goldstone, R. L. (2006). The interplay between perceptual organization and categorization in the representation of complex visual patterns by young infants. Journal of Experimental Child Psychology, 95, 117-127.

Siddiei, K., Tresness, K. J., \& Kimia, B. B. (1996). Parts of visual form: Psychophysical aspects. Perception, 25, 399-424.

Singh, M., \& Hoffman, D. D. (2001). Part-based representations of visual shape and implications for visual cognition. In T. F. Shipley \& P. J. Kellman (Eds.), Advances in psychology: Vol. 130. From fragments to objects: Segmentation and grouping in vision (pp. 401-459). New York: Elsevier.

Singh, M., Seyranian, G. D., \& Hoffman, D. D. (1999). Parsing silhouettes: The short-cut rule. Perception \& Psychophysics, 61, 636660 .

Snodgrass, J. G., \& VANDERWART, M. (1980). A standardized set of 260 pictures: Norms for name agreement, image agreement, familiarity, and visual complexity. Journal of Experimental Psychology: Human Learning \& Memory, 6, 174-215.

XU, Y., \& Singh, M. (2002). Early computation of part structure: Evidence from visual search. Perception \& Psychophysics, 64, 10391054.

Younger, B. A., \& Fearing, D. D. (1999). Parsing items into separate categories: Developmental change in infant categorization. Child Development, 70, 291-303.

(Manuscript received August 8, 2009; revision accepted for publication January 11, 2010.) 